\title{
Desarrollo del lenguaje y preparación para la lectura en Educación Infantil
}

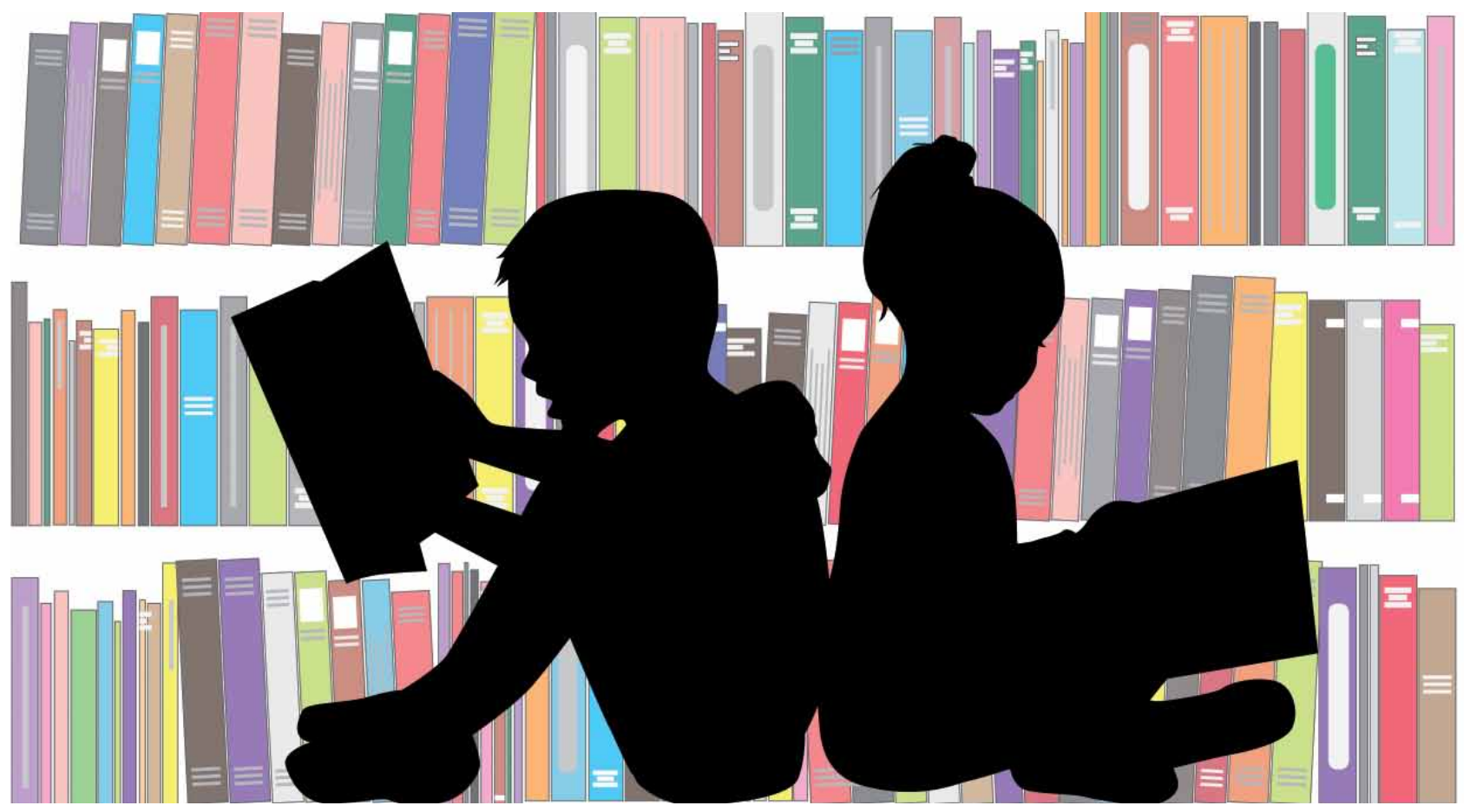

Aprender a leer es uno de los logros más importantes en la vida escolar. Padres y educadores observan con atención dicho proceso. La continuidad entre el lenguaje oral y escrito posibilita que incorporemos la promoción de la 'conciencia fonológica' en prelectores, mediante programas de actividades lingüísticas lúdicas, para prevenir

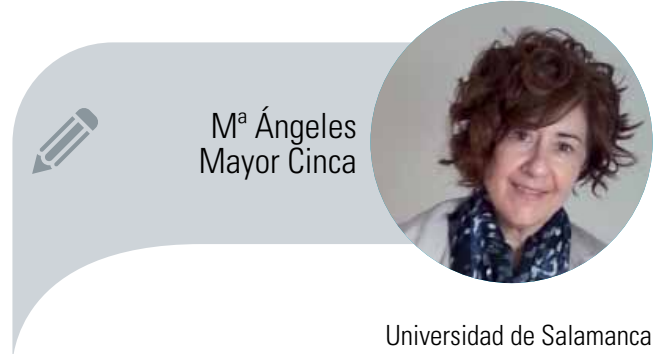
mayor@usal.es

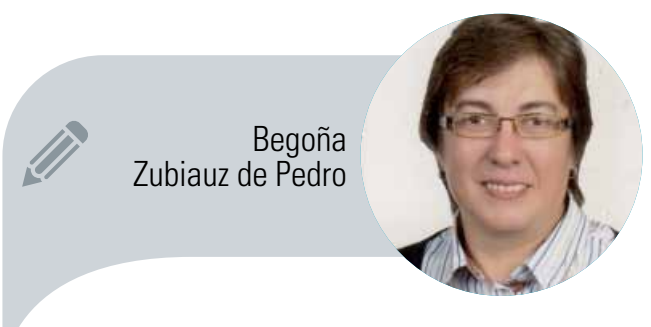

Universidad de Salamanca bzubiauz@usal.es posibles dificultades. 


\section{La articulación entre el lenguaje oral y escrito}

El proceso inicial de aprender a leer resulta muy costoso para gran cantidad de alumnos y árido para muchos de ellos. Probablemente, se deba al hecho de que la lectura, es decir, la comprensión de las palabras y posteriormente del texto, requiere integrar dos tipos de habilidades: (a) una adecuada comprensión (y comunicación) del lenguaje oral, previa a la lectura y (b) la capacidad de reconocer palabras escritas, que exigirá llevar a cabo ciertas operaciones nuevas y específicas (MAYOR, ZUBIAUZ y DíEZ-VILLORIA, 2005).

Algo que suele comprobarse, sin duda, es que en estos inicios del aprendizaje de la lectura aparecen diferencias individuales evidentes entre los pequeños, aspecto que es muy relevante, y al que hemos de prestar atención si consideramos, sobre todo, el escaso tiempo que el currículum escolar concederá, en su momento, para el aprendizaje formal del lenguaje escrito. Y desde luego, aquellos niños que hayan venido manifestado alguna dificultad para lograr una adecuada comunicación oral, muy probablemente requerirán una enseñanza de la lectura adaptada a sus necesidades, y a los procesos demorados, o divergentes, mediante los que han ido estableciendo su particular comunicación.

Desde hace ya unos cuantos años, disponemos de un adecuado modelo explicativo sobre los procesos necesarios para que los escolares aprendan a leer correctamente. Y concretamente, en relación al tema que nos ocupa, desde el modelo psicolingüístico de la adquisición de la lectura (Alegría, 2006) se ha comprobado, de forma consistente, la relación temprana entre lectura y conciencia fonológica. Hemos de señalar que esta habilidad es muy específica, y que requiere que los niños lleguen a ser capaces de operar con los segmentos de la palabra que tienen entidad lingüística (rima, sílaba y fonema), y de realizar con rapidez tareas tales como encadenar, añadir, quitar o sustituir sonidos, etc. con dichos segmentos. Desde esta corriente psicolingüística existen numerosas investigaciones que demuestran que la instrucción explícita en las tareas mencionadas mejora, significativamente, la lectura de los alumnos que están aprendiendo a leer (AdAms, 1990; Zubiauz y MAYOR, 2008) y, más aún, la del alumnado con dificultades para su aprendizaje (BRYANT Y BRADLEY, 1998; RuEDA, 1995).

Por lo tanto, podemos afirmar que el aprendizaje de la lectura conlleva procesos como los mencionados, que son de una alta especialización (la llamada "conciencia fonológica") y que habrán de automatizarse en apenas un curso escolar, espacio que establece el currículum para lograr el aprendizaje de la lectura al iniciarse Primaria. Hemos de considerar también que a esa misma edad (en torno a los 6 años), se está afianzando el desarrollo lingüístico oral de los niños y niñas, en los diferentes procesos (fonológico, morfosintáctico, léxico-semántico y pragmático), tal y como reflejan sus producciones conversacionales. Este momento, por tanto, al ser tan esencial del desarrollo comunicativo lingüístico, requiere que lo abordemos analizando las implicaciones de la articulación entre lenguaje oral y escrito con el fin de definir una óptima enseñanza de la lengua.

Por todo ello, desde el contexto escolar y antes del inicio formal de la lectura, debemos poner en práctica programas

Figura 1. Conciencia fonológica

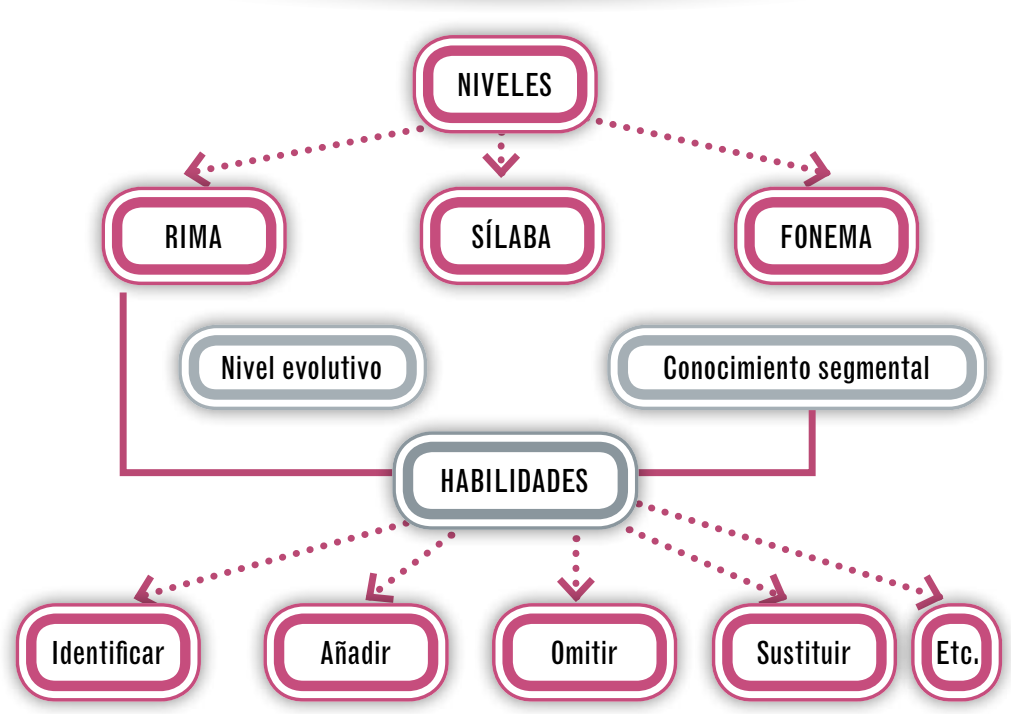


Figura 2. La enseñanza de la lectura en Europa (Eurydice, 2011)

Cinco claves sobre el comienzo de la lectura en la Educación Infantil

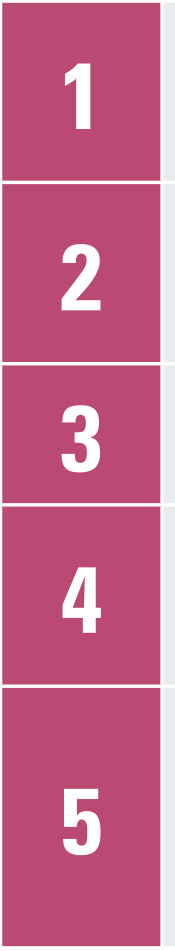

"La fase en la que se ponen los cimientos del desarrollo lector es una etapa crucial en la adquisición de la lectura. Las dimensiones que más influyen en las etapas tempranas del desarrollo lector son: la conciencia fonológica y fonemática, la fonética y la fluidez verbal".

"La conciencia fonológica es la habilidad para detectar y manipular los segmentos sonoros del lenguaje oral. La conciencia fonemática, es decir, la comprensión de los fonemas, es un componente fundamental de la conciencia fonológica".

"La incorporación de estrategias para aumentar la conciencia fonológica en las clases de infantil es de crucial importancia para el éxito de los niños en la adquisición de la lectura".

“La mayoría de las investigaciones recalcan la necesidad de enseñar la fonología de forma sistemática y de acuerdo a un plan o programa claro, en lugar de prestar atención solo de manera esporádica a la correspondencia grafía-fonema".

“En general, es importante recordar que aunque la conciencia fonológica, la conciencia fonemática, la enseñanza de la fonología y la fluidez se han convertido en los métodos de enseñanza más populares, más utilizados y sobre los que más se ha investigado, no son los únicos. Estos métodos sólo abarcan algunos de los procesos que, en conjunción con otros, son necesarios para que un alumno llegue a leer".

que incorporen al aprendiz a actividades relativas al reconocimiento de la rima, el análisis y la combinación de segmentos lingüísticos, como la sílaba y, muy especialmente, el fonema. Esta sensibilización, de hecho, puede y debería alcanzarse antes de que el alumnado comience a leer de forma explícita, y siempre mediante actividades lúdicas, puesto que el descubrimiento del carácter alfabético de nuestro sistema de notación (caracterizado por la correspondencia exacta entre grafema y fonema) agradece la familiarización que los juegos de lenguaje ofrecen a los niños, a la hora de identificar el carácter arbitrario, y por tanto difícil de alcanzar, de dicha correspondencia.

Es indudable que la relación entre la conciencia fonológica y la lectura es bidireccional, aunque la meta que aquí nos proponemos es definir únicamente el camino de ida, dotando a los escolares de las estrategias necesarias que le habiliten en el autoaprendizaje de la lectura (SHARE, 1995), aunque con las ayudas pertinentes. En ese sentido, hemos de reiterar que lo relevante en la construcción de esa direccionalidad implica, sobre todo, afianzar el desarrollo del lenguaje oral mediante actividades de segmentación que permitan al aprendiz el descubrimiento de las reglas de correspondencia sonido-articulación-letra, sin apenas percatarse, es decir,jugando a romper y ensamblar fragmentos de palabras. Por ello, probablemente, la pregunta de carácter práctico que se plantea lógicamente el profesorado es: ¿cómo enseñar eficazmente esas
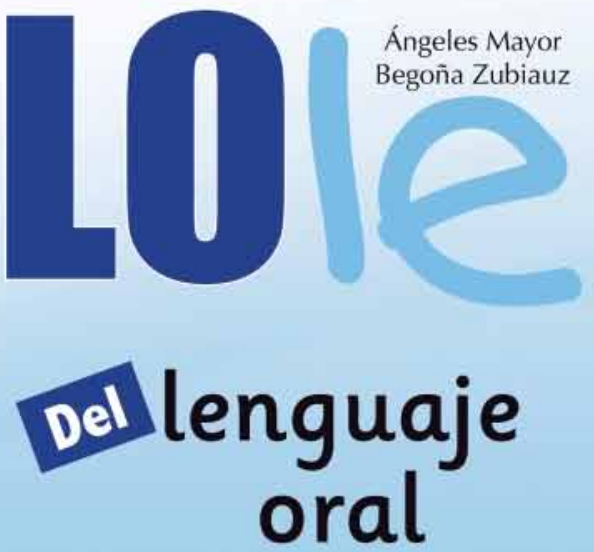

a) lenguaje escrito

A la vanguardia de la evaluación psicológica
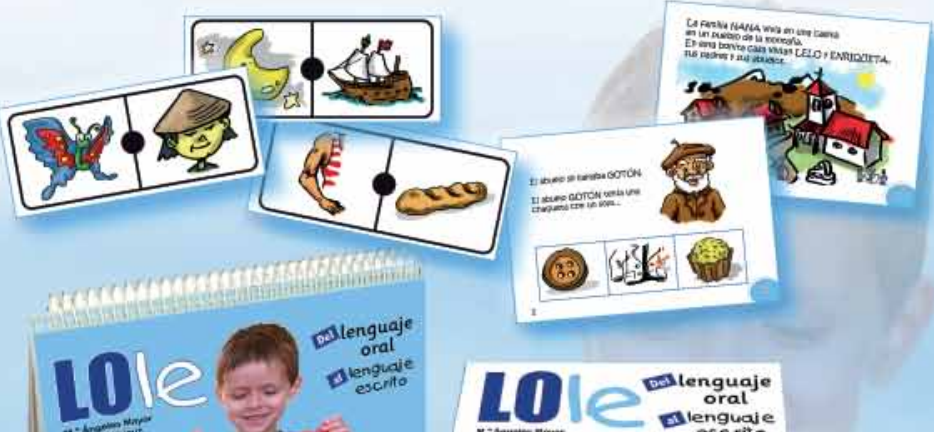

$112 \begin{gathered}\text { alenguaje } \\ \text { oral } \\ \text { anguaje } \\ \text { escrito }\end{gathered}$
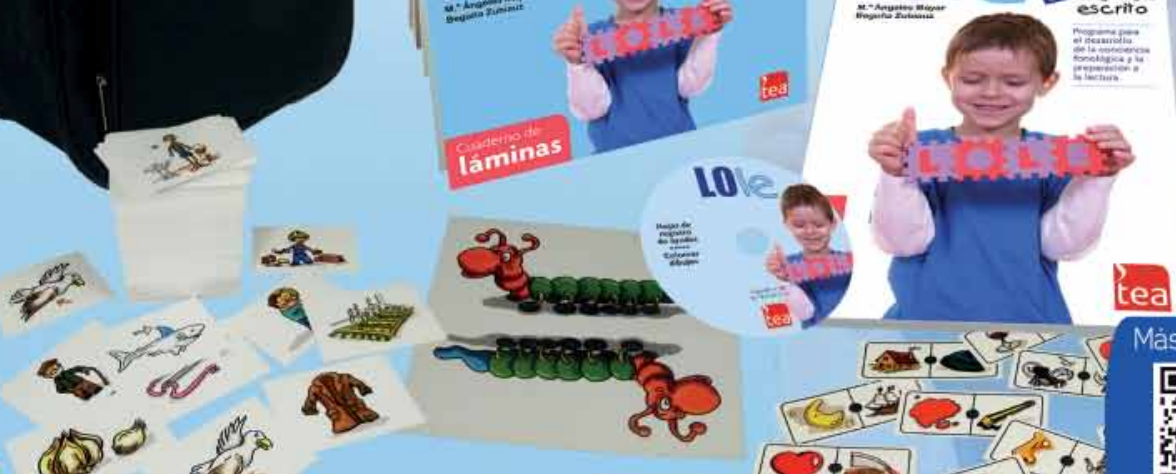

Q
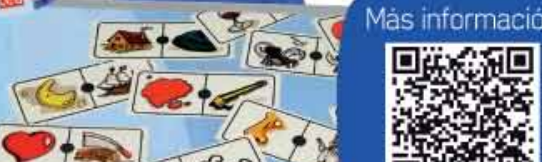

5 o tैh-50 
Figura 3. Modelo autoaprendizaje de la lectura

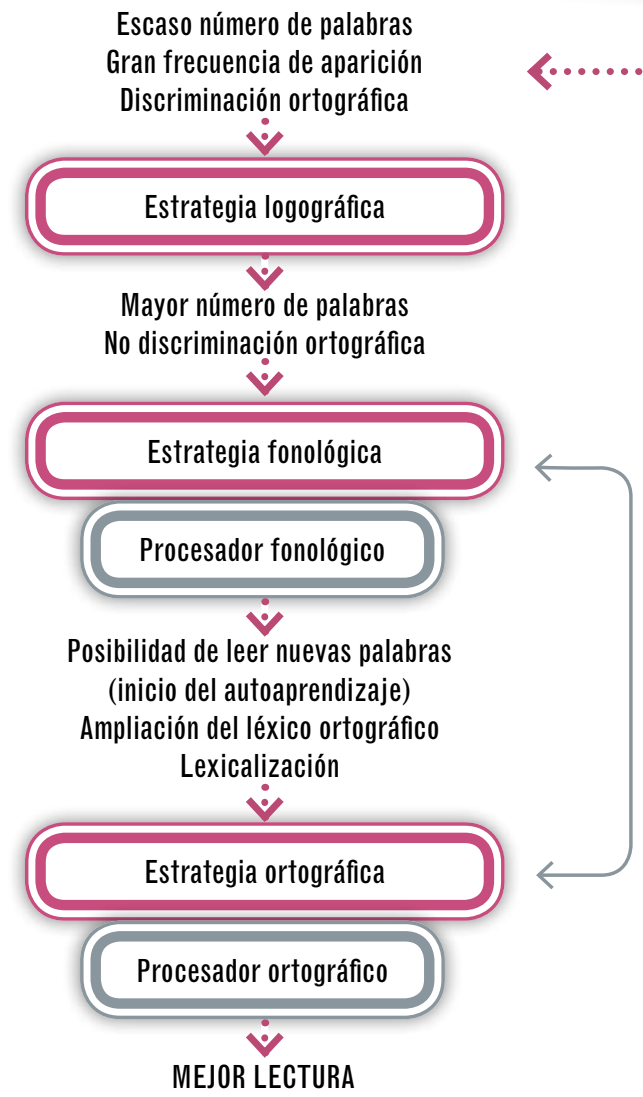

nuevas habilidades, tan esenciales para leer correctamente? Es lo que trataremos de responder a continuación.

\section{Facilitando el aprendizaje inicial} de la lectura desde el lenguaje oral

Leer implica, al menos en un primer momento, reconocer palabras. Las primeras palabras que reconocen los niños se caracterizan por su frecuente aparición en contextos cotidianos (ej. marcas de alimentos), y que identifican mediante las características que rodean a las palabras (gráficas/contextuales).

Ahora bien, en un segundo momento, se requerirá un procesamiento más complejo. Podemos distinguir dos estrategias para leer una palabra y descubrir su significado: la estrategia fonológica y la estrategia lexical (CUETos, 2008). En el inicio del proceso de aprendizaje de la lectura, como ya hemos mencionado anteriormente, el alumno ha de alcanzar el dominio del principio alfabético, es decir, dominar las reglas de correspondencia grafemafonema. Este principio es esencial para poder leer y escribir palabras nuevas (ej., electricista) con las que el aprendiz no
INPUT ESCRITO

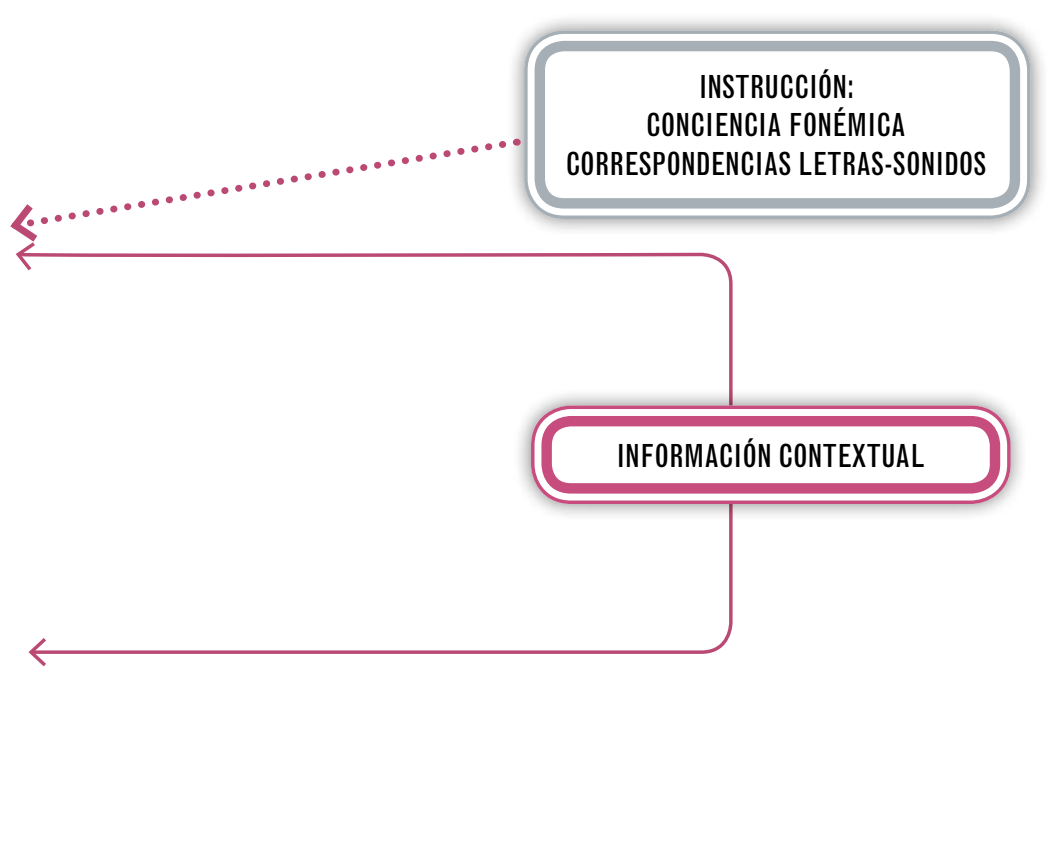

Figura 4

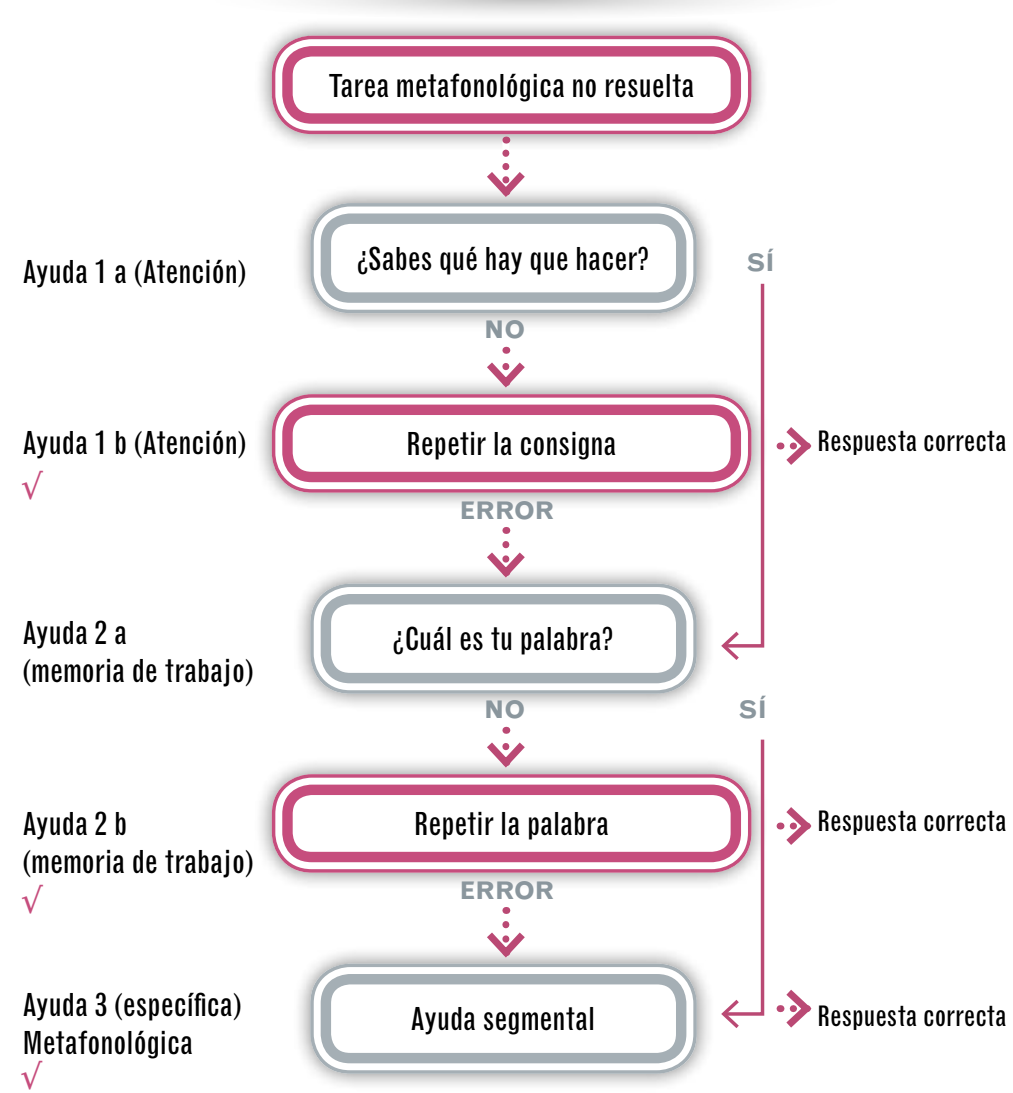




\section{El programa "LOLE -Del lenguaje oral al lenguaje escrito"}

El programa "LOLE -Del lenguaje oral al lenguaje escrito" toma su nombre de su objetivo principal: desarrollar las relaciones existentes entre los niveles linguísticos oral y escrito, estableciendo un puente entre ambos, mediante el desarrollo de la conciencia fonológica.

\section{Objetivos}

(a) Optimizar el aprendizaje inicial de la lectura en alumnos prelectores mediante actividades de lenguaje oral.

(b) Identificar dificultades en escolares de Educación Infantil que propicie una actuación preventiva para los alumnos con posibles dificultades de aprendizaje en Educación Primaria.

Aplicabilidad en las aulas de Infantil: aspectos base

У 32 unidades didácticas que hacen posible un trabajo sistemático durante al menos un curso escolar, que puede ampliarse dependiendo de la edad y el ritmo de aprendizaje;

$\searrow$ carácter oral: garantiza que las tareas de segmentación se inserten dentro del contexto de las actividades conversacionales que el profesor adapta al grupo, a partir del manual del programa en el que se describe detalladamente el procedimiento de aplicación para cada unidad;

\ carácter lúdico: el material gráfico de apoyo (tarjetas coloreadas, dominó de palabras, láminas con escenas, etc.) permite asegurar una atención y motivación óptimas;

\ selección de palabras basadas en el vocabulario infantil: se recogen todas las combinaciones de fonemas en español, haciendo especial énfasis en las combinaciones más complejas (ej. CCV).

$\searrow$ ofrecimiento de ayudas por parte del maestro y su evaluación individualizada, mediante las hojas de registro en las que se recogen el tipo de ayuda prestado y la respuesta del niño, lo que permite construir un perfil de aprendizaje individual y grupal, que resulta de gran utilidad para el profesorado, especialmente para identificar a los alumnos con mayores dificultades en el aprendizaje.

\section{Estructura del LOLE}

El Programa incluye 32 unidades didácticas en las que se trabajan diferentes niveles (rima, sílaba y fonema) y tareas (identificación, adición y omisión) de conciencia fonológica, dispuestas de menor a mayor dificultad. Estas unidades se distribuyen en dos módulos: el módulo I se centra en los niveles de rima y sílaba (unidades 1 a 16) y el módulo 2 en el nivel de fonema (unidades 17-32).

Las actividades han sido diseñadas para que sean aplicadas por el profesor dentro de su grupo-clase, aunque también pueden aplicarse de manera individual. Por otro lado, todas las actividades siguen el mismo guión que es el siguiente: Objetivo de la actividad - Habilidad de conciencia fonológica que se trabaja - Material de apoyo (palabras a trabajar) - Procedimiento de aplicación - Ayudas a proporcionar.

está familiarizado ortográficamente. Así, la primera vez que intente leer la palabra "electricista" no podrá hacerlo mediante un reconocimiento gráfico, sino que tendrá que romper la secuencia ortográfica para reconvertir sus elementos (letras), uno a uno, en sus sonidos equivalentes (por medio del sistema fonológico del lenguaje oral), y ensamblar luego esa secuencia de sonidos para reconocer la palabra como un todo y acceder a su significado. Este procedimiento para reconocer palabras se denomina vía indirecta o fonológica. Su dominio exige disponer de un sistema fonológico oral plenamente funcional y rápido (para manejar la correspondencia sonidoarticulación), gracias al cual se consigue el proceso de recodificación mencionado.

En cambio, cuando el niño ha de leer una palabra muy familiar para él (ej. perro), es muy probable que pueda reconocerla ortográficamente de forma inmediata, si la ha visto escrita muchas veces, activando casi simultáneamente la secuencia auditiva correspondiente y el significado al que se refiere. Este procedimiento de lectura de palabras es la denominada vía directa o léxica. Lógicamente, los lectores competentes emplean ambas vías de forma simultánea y eficiente, dependiendo de la necesidad de leer palabras conocidas o nuevas.

Es evidente también que el progreso en el aprendizaje de la lectura y, en concreto, la comprensión lectora, implicarán procesos posteriores y más complejos que el reconocimiento de palabras. Sin embargo, no olvidemos que la mayor efectividad a la hora de reconocer palabras, convirtiendo grafemas en fonemas, explica parte de las diferencias que se observan en la comprensión de un texto, lo que demuestra que es un logro esencial. Más aún, la automatización de ese reconocimiento de palabras, o la rapidez con que un lector experto aplica las habilidades relacionadas, es básica para poder dedicar la mente a la interpretación del texto en función de los propios conocimientos, ya que nos permite liberar recursos cognitivos básicos para ponerlos a disposición de los procesos de más alto nivel, exigidos por la comprensión. Esa automatización es esencial, y lo es hasta tal punto que, paradójicamente, acaba pareciendo insignificante una vez se ha conseguido. De ahí la importancia 
de instruir en el momento oportuno, con el procedimiento adecuado, y ajustándonos a las características de cada escolar.

El objetivo, por tanto, es que todos los niños consigan alcanzar ese dominio automático antes de enfrentarse al aprendizaje de la lectura, que reciban las ayudas que necesiten individualmente, y que consigan ese descubrimiento disfrutando de situaciones conversacionales con el grupo clase.

Esta adquisición de nuevos contenidos requiere también que el educador establezca una relación interpersonal idónea con el aprendiz, dentro de un espacio cooperativo, en el que construya una guía de actuación, para ofrecer a cada uno la ayuda precisa, asegurándose de estar trabajando dentro de la "zona de desarrollo próximo" (VYGOTSKI, 1964) del alumno, es decir, ayudándole a utilizar procedimientos de los que ya dispone, pero que por sí solo no utilizaría. El préstamo de estas ayudas debe ser inversamente proporcional a la competencia del alumno y el número de las mismas se ha de ir disminuyendo o retirando, a medida que éste asuma el control de la actividad. El establecimiento de esa relación interpersonal idónea requiere que el alumnado conozca la meta del aprendizaje, a fin de que valore sus progresos y se independice progresivamente. En otras palabras, el educador debe guiar la actividad del alumno, super-

\section{paria Saber mấS}

Mayor, Ma A. y Zubiauz, B. (2011). LOLE: Del lenguaje oral al lenguaje escrito. Madrid: TEA Ediciones.

Defior, S. y Serrano, F. (2011). La conciencia fonológica, aliada de la adquisición del lenguaje escrito. Revista de Logopedia, Foniatría y Audiología, 31(1) 2-13

Gellert, A. S. y ElBro, C. (2015) Does a Dynamic Test of Phonological Awareness Predict Early Reading Difficulties? A Longitudinal Study From Kindergarten Through Grade 1. Journal of Learning Disabilities DOI: 10.1177/0022219415609185

\section{ÁQORA DE PROFESORES}

La importancia de las ayudas en la evaluación del proceso de desarrollo de las habilidades metafonológicas (evaluación dinámica)

En el programa "LOLE" se distinguen dos tipos de ayudas: ayudas generales (ayuda 1 y 2) y ayudas específicas (ayuda 3), Las ayudas generales permiten asegurarnos de que el grado de atención y la activación de la memoria de trabajo, necesarios para la resolución de las actividades, son los adecuados. La ayuda específica se encamina a facilitar al alumno la puesta en marcha de la habilidad fonológica que se practica en la actividad.

$1^{\text {a }}$ ayuda. Recuerdo de la tarea. Esta ayuda tiene por objeto asegurarse de que el alumno sabe lo que ha de hacer, es decir, que está en situación de participar activamente en la interacción. La ayuda admite dos grados: a) preguntar por la tarea (menor ayuda); y b) repetir la consigna (mayor ayuda). La ayuda a) pretende que el alumno demuestre que conoce lo que se le pide que haga, de modo que si responde adecuadamente no se utilizará la ayuda b) y se continuará con la ayuda 2. Se empleará la ayuda b) cuando el alumno no responda 0 cuando explique inadecuada- mente la tarea que se le demanda.

$2^{\mathrm{a}}$ ayuda, Recuerdo de la palabra. Esta ayuda permite al educador asegurarse de que el alumno está centrado en la tarea. Puede suceder que una vez que ya se ha comprobado que el niño sabe lo que ha de hacer, se observe que ha olvidado la palabra o segmento con el que ha de realizar la tarea que se le pide. De nuevo contamos con dos grados:

a) Preguntar por la palabra/sílaba/fonema y b) Repetir el elemento linguíístico. La ayuda a) tiene por objeto dar la oportunidad al alumno de demostrar que sabe cuál es "su" palabra. Si el niño responde correctamente continuaremos con la ayuda 3. En caso contrario, emplearemos la ayuda b) y le diremos de nuevo al alumno la palabra 0 segmento con el que tiene que trabajar.

$3^{a}$ ayuda: Específica. Esta ayuda se denomina así porque está dirigida, en concreto, al desarrollo de la conciencia fonológica, es decir, va destinada a facilitar la realización de las diversas tareas que contempla el programa "LOLE": reconocimiento de la rima, identificación, adición y omisión (en posiciones inicial y final) tanto de sílaba como de fonema. En este caso, se trata de que el alumno "construya" una representación alternativa a la lingüística que, dependiendo de la ayuda, puede referirse a la palabra (o segmento) de partida que recibe el alumno, al segmento con el que ha de operar (identificar, omitir 0 añadir), e incluso a la palabra que constituye la solución. Esa representación, de naturaleza más concreta ( $p$. ej., visual), permite establecer un paso intermedio que permite lograr la manipulación de las unidades linguíísticas, de carácter mucho más abstracto.

visar su ejecución, compartir la meta, transferir progresivamente el control, prestar las ayudas oportunas y retirarlas adecuadamente (BrAvo, 2004) •

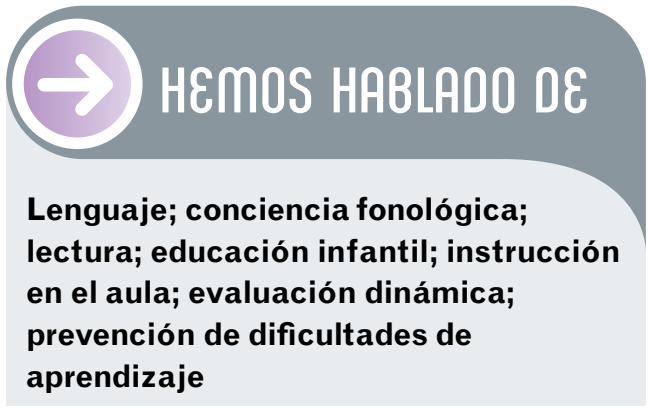

Este artículo fue solicitado por PADRES y MAESTROS en septiembre de 2015, revisado y aceptado en enero de 2016. 


\section{¿Cómo orientar profesionalmente a tu hijo? \\ Manual práctico para padres}

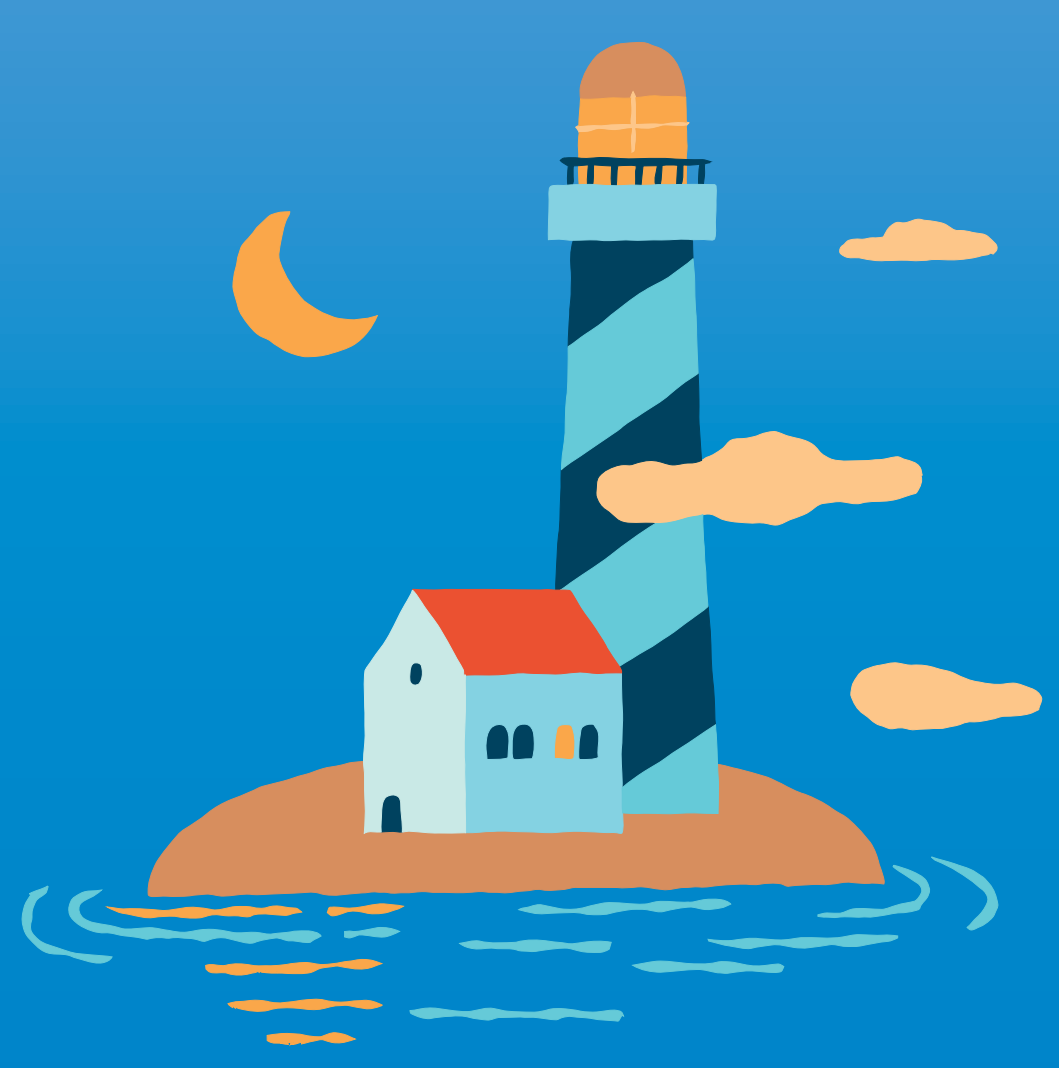

Vicente Hernández Franco (Coord.)

Fundación Bertelsmann

El objetivo principal que tiene esta guía práctica de Orientación Profesional para padres es ofrecer a las familias la información relevante para que puedan formarse su propio criterio a la hora de entender, enfocar y asesorar, desde la responsabilidad parental, todo el proceso de

Orientación Profesional de sus hijos.

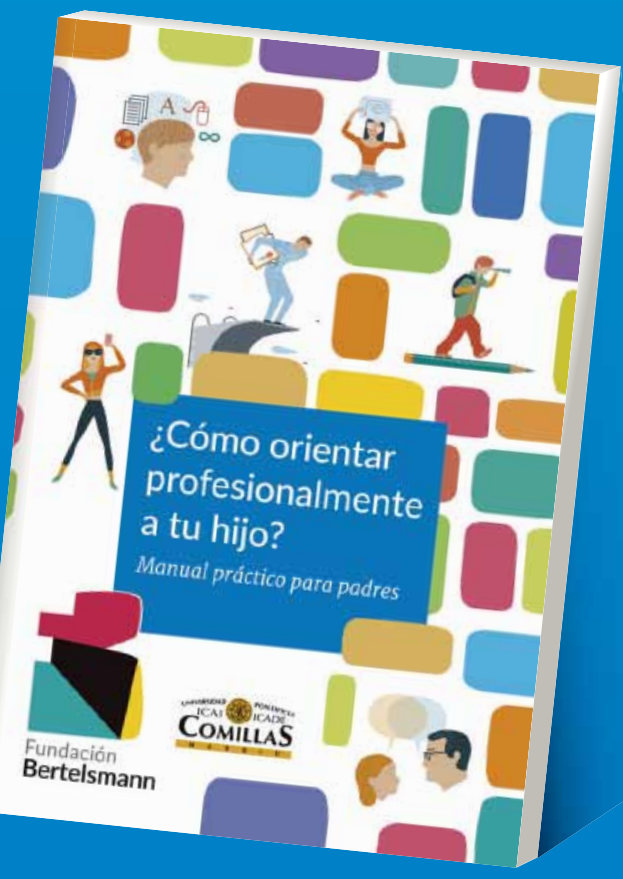

Por el empleo juvenil

Puedes descargar gratuitamente el libro

Tel.: +34932687373 en versión pdf: https://goo.gl/RMcZIR

info@fundacionbertelsmann.org

Cómo seguirnos

www.fundacionbertelsmann.org

@FBertelsmann

www.facebook.com/FundacionBertelsmann

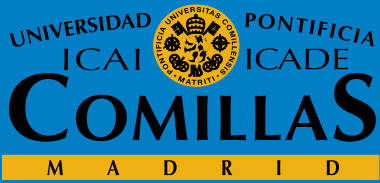

